\title{
Autism, Early Psychosis, and Social Anxiety Disorder: a transdiagnostic examination of executive function cognitive circuitry and contribution to disability
}

\author{
Eleni A. Demetriou', Christine Y. Song ${ }^{1}$, Shin H. Park ${ }^{1}$, Karen L. Pepper ${ }^{1}$, Sharon L. Naismith ${ }^{2,3}$, Daniel F. Hermens ${ }^{2}$,
} lan B. Hickie ${ }^{1,2}$, Emma E. Thomas', Alice Norton', Django White ${ }^{2}$ and Adam J. Guastella,2

\begin{abstract}
The disability burden in clinical cohorts with social impairment is significant, leading to poor functional outcomes. Some of this impairment has been linked to executive dysfunction. In this study, a transdiagnostic approach was taken to identify executive function (EF) processes in young adults that may underpin social impairment and to evaluate their contribution to disability. Comparisons were made between three prominent disorders that are characterized by social impairments, Autism Spectrum Disorder (ASD), Early Psychosis (EP) and Social Anxiety Disorder (SAD), as well as a neurotypically developing group (TYP). We examined whether overall disability could be predicted by neuropsychological and self-report assessments of EF. Our study showed that ASD participants demonstrated impaired performance on most domains of EF compared to the TYP group (mental flexibility, sustained attention and fluency) while the EP group showed impairment on sustained attention and attentional shifting. The SAD participants showed EF impairment on self-report ratings, even though their objective performance was intact. Self-reports of EF explained a significant percentage (17\%) of disability in addition to the variance explained by other predictors, and this was particularly important for ASD. This is the first study to compare EF measures across clinical groups of social impairment and suggests unique cognitive-circuitry that underpins disability within groups. Impairments in EF were broad in ASD and predicted disability, EP impairments were specific to attentional processes and SAD impairments likely relate to negative self-monitoring. Self-report, as opposed to performance-based EF, provided best capacity to predict disability. These findings contribute to transdiagnostic circuitry models and intervention strategies.
\end{abstract}

\section{Introduction}

The disability burden in clinical cohorts with social impairment is significant and typically associated with poor functional outcomes ${ }^{1-3}$. Social impairment and disability have been linked to poor executive function (EF) performance $^{4,5}$ in these groups and EF may be a useful

Correspondence: Adam J. Guastella (adam.guastella@sydney.edu.au) ${ }^{1}$ Autism Clinic for Translational Research, Brain and Mind Centre, Central Clinical School, Faculty of Medicine, University of Sydney, Camperdown 2050, Australia

${ }^{2}$ Youth Mental Health Unit, Brain and Mind Centre, Central Clinical School, Faculty of Medicine, University of Sydney, Camperdown 2050, Australia Full list of author information is available at the end of the article. cognitive marker to predict disability. Assessment of EF is traditionally based on neuropsychological (objective) measures of the level of performance across cognitive domains $^{6}$. More recently, standardised scales of self/ informant (subjective) based ratings of EF have been introduced with empirical support that these may be more ecologically valid assessments of $\mathrm{EF}^{7}$. Understanding how $\mathrm{EF}$ and underlying cognitive circuitry may contribute to disability in clinical groups with social impairment and, more specifically, ascertaining the contributions of objective and subjective measures of EF may be pivotal for diagnosis and functional outcomes. Such research is

\section{(c) The Author(s) 2018}

(c) (i) Open Access This article is licensed under a Creative Commons Attribution 4.0 International License, which permits use, sharing, adaptation, distribution and reproduction cc) in any medium or format, as long as you give appropriate credit to the original author(s) and the source, provide a link to the Creative Commons license, and indicate if changes were made. The images or other third party material in this article are included in the article's Creative Commons license, unless indicated otherwise in a credit line to the material. If material is not included in the article's Creative Commons license and your intended use is not permitted by statutory regulation or exceeds the permitted use, you will need to obtain permission directly from the copyright holder. To view a copy of this license, visit http://creativecommons.org/licenses/by/4.0/. 
particularly important in early adulthood ${ }^{8}$ when brain development and transition into higher education, work, and adult social relationships coincide with establishing lifelong roles.

Autism Spectrum Disorder (ASD), Psychotic Disorders and Social Anxiety Disorder (SAD) are the three most common and recognised conditions characterized by social impairment. Traditionally, the influence of EF has been examined within disorders. Numerous researchers have proposed common aetiologies and maintaining factors underpinning disability in these cohorts, raising potential for common circuitry processes across psychiatric disorders that may predict disability ${ }^{9,10}$. In the neurodevelopmental cluster (i.e. ASD, Psychosis) EF is believed to result from differential brain development ${ }^{11}$ which may contribute to disability ${ }^{12}$. Empirical support for a link between EF and social impairment has been reported for $\mathrm{ASD}^{4,13}$ and psychotic disorders ${ }^{14}$, including the most common psychotic presentation in young adults, Early Psychosis (EP). These EF impairments may contribute to poor functional outcomes ${ }^{14,15}$. Neural circuitry studies show involvement of the prefrontal cortex (PFC) and, in particular, the dorsolateral and ventrolateral prefrontal cortex (DLPFC, VLPFC) in EF performance for both schizophrenia and ultra-high risk psychotic populations ${ }^{16,17}$. Within ASD, a recent meta-analysis suggested more global executive dysfunction ${ }^{18}$ with little evidence of selective dysfunction in specific EF domains (although few studies examined adult samples). Similarly, the underlying brain circuitry in ASD suggests widespread functional and anatomical differences ${ }^{19}$, although neuroimaging studies have also identified specific deficits in the $\mathrm{PFC}^{20}$ and fronto-striatal circuitry ${ }^{21}$. To date however, the relationship of disability to EF has been explored by few studies ${ }^{22}$ and mostly in children or young adolescents ${ }^{13,23}$. There has also been no transdiagnostic examination of the influence of EF to disability in neurodevelopmental cohorts.

Where the primary presenting condition is SAD, the relationship between neuropsychological difficulties and disability is more tenuous ${ }^{5,24}$. Mixed results have noted potential deficits in mental flexibility, verbal fluency ${ }^{24}$ and in the areas of sustained attention and concept formation $^{5}$. There is no clear empirical support, however, for impaired neural circuitry specific to EF in SAD. For SAD, underlying aetiology and maintaining factors are believed to focus primarily on fear circuitry ${ }^{25}$, maladaptive cognitions, negative evaluation ${ }^{26}$ and avoidance ${ }^{27}$. Research suggests that these may be modulated by impaired topdown connectivity between the ventromedial prefrontal cortex (VMPFC), the amygdala ${ }^{28}$ and aberrant processing in the default mode network (DMN), leading to disturbed self-evaluative and self-referential processing ${ }^{28}$. Significant impairment in social functioning has been reported in $\mathrm{SAD}^{27}$ however, the relationship between $\mathrm{EF}$ and disability in SAD has not been specifically studied.

\section{Study aims}

In this study, we aim to address the relationship between EF and disability in young adults with disorders characterized by social impairment. As far as we are aware, no study has yet compared EF performance across these disorders. The first aim of this study was to determine EF outcomes in treatment seeking young adults with presenting primary diagnosis of ASD, SAD and EP, and a neurotypical control group. It was predicted that young adults diagnosed with ASD and EP would show broad EF impairments in comparison to those with SAD and neurotypical controls. The second aim of this study was to evaluate the predictive value of EF to disability across and within these cohorts. It was predicted that EF would predict disability for participants with ASD and EP, but not SAD. Finally, we were interested in the degree that performance measures or self-report measures predicted disability.

\section{Method \\ Participants}

The University of Sydney Ethics Committee approved the research protocol (No. 2013/352) for this project and informed consent was obtained directly from each participant prior to inclusion in the study. A cohort of young adults $(N=253$; Age: $\mathrm{M}=23.16, \mathrm{SD}=5.80)$ presenting for treatment and/or social skills development at the Autism Clinic for Translational Research (ACTr) and Headspace Brain and Mind Centre clinics, were recruited into the study. Participants met criteria for the primary presenting disorder of either ASD, $N=60 ; \mathrm{EP}, N=58$; or SAD, $N=76$. Clinical diagnoses were made by qualified clinicians at the ACTr, based on standardised clinical interviews and diagnostic assessment instruments. Neurotypical control participants (TYP; $N=59$ ) were recruited through advertising at a number of different university websites. All participants were screened and excluded from the study if they had intellectual disability $(\mathrm{IQ}<70)$ or current substance dependence. The TYP participants were excluded if they reported a mental health diagnosis (past or current), were currently experiencing significant levels of depression, anxiety or stress (DASS-21), or if they scored at clinical cut-offs for SAD (SIAS) and/or ASD $(\mathrm{AQ})$.

\section{Measures}

A detailed description of all measures is provided in Supplementary Table 1 . The assessment battery comprised of a combination of diagnostic assessments, neuropsychological tests and self-report measures of EF, mood and disability. Diagnostic assessments consisted of 
standardised clinical interviews for the diagnosis of ASD (ADOS), EP (SCID-I) and SAD (ADIS), psychotic symptoms were assessed with the SAPS and SANS scales. Neuropsychological tests comprised of assessments of pre-morbid IQ (WTAR) and performance measures of EF assessing the domains of 'Set Shifting' (CANTAB-IED), 'Mental Flexibility' (TMT-B) and 'Verbal Fluency' (COWAT). The cognitive domains of 'Sustained Attention' (CANTAB-RVP) and 'Psychomotor Speed' (TMT-A) were also assessed. Self-report ratings of EF and disability were based on the BRIEF and WHODAS respectively. Symptom severity was assessed by the DASS-21, AQ and the SIAS.

\section{Data analysis}

Data analysis was performed using the IBM SPSS Statistics version 24. Univariate (ANOVA) and Multivariate Analysis of Variance (MANOVA) examined differences between the groups on EF and disability. Two-sided tests with Bonferroni confidence interval adjustments were made for all statistical comparisons, alpha was set at $p=$ 0.01 to control for multiple comparisons. Multiple Regression (MR) analyses examined the predictive value of the EF measures on disability. The EP group did not complete the BRIEF and MR analysis for this group were based on performance only measures of EF. For each of the MR analyses, IQ, Education, Depression and EF performance measures were entered in Step 1 and where available, the BRIEF was entered in Step 2.

\section{Results}

\section{Statistics}

Sample size was based on the number of participants tested at the conclusion of the study. Our sample size per group exceeded the suggested minimum $(n=30)^{29}$ for violations against the assumptions of normality and equality of variance in MANOVA. The MANOVA was carried out with and without the two covariates (IQ, Education) and the statistical outcomes were comparable. Results are reported for the analysis including covariates. All assumptions for MR were met with the exception of multivariate outliers as assessed by Mahalanobis Distance. An examination of the largest outliers together with the Statistic for Cook's Distance indicated that they do not exert an influence on the model ${ }^{29}$ and no further action was taken.

\section{Demographics}

Participant demographic information is presented in Table 1. No significant gender differences were observed between the diagnostic and control groups, $\chi^{2}(3, N=253)=$ 2.73, $p>0.05$. The overall significant effect for Age $\left(F_{3,249}\right.$ $=4.33, p=0.005)$ did not hold for pairwise comparisons. Significant overall effects were observed for Education

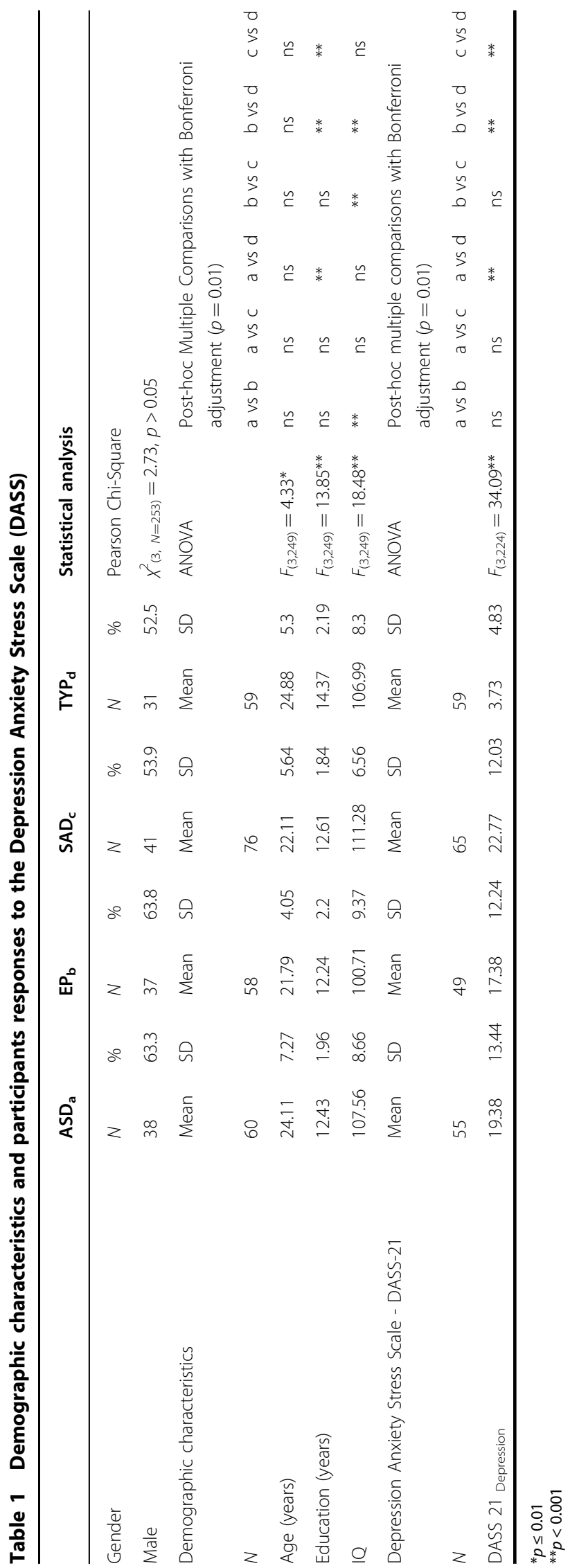



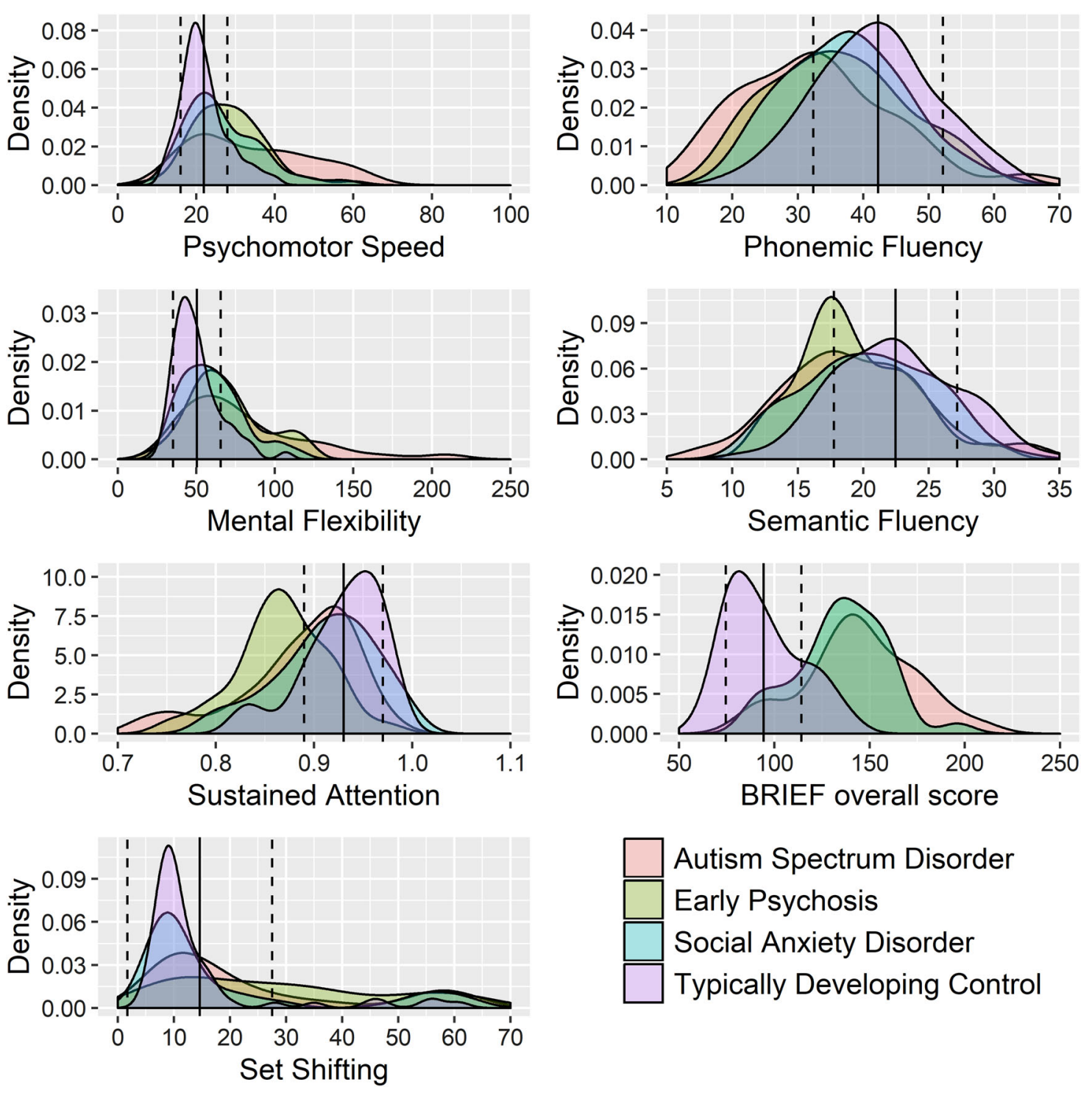

Fig. 1 Response distribution of EF neuropsychological and self-report measures

$\left(F_{3,249}=13.85, p<0.001\right)$, IQ $\left(F_{3,249}=18.48, p<0.001\right)$ and Depression $\left(F_{3,224}=34.09, p<0.001\right)$. Pairwise comparisons showed that: the TYP group had significantly more years of education compared to each of the clinical groups; the EP group had significantly lower IQ compared to each of the other groups. The clinical groups reported higher levels of depression compared to the TYP group with no differences between the clinical groups.

\section{Group comparison of EF performance/self-report measures and disability self-report measures}

For the distribution of participant responses for the neuropsychological and self-report measures of EF refer Fig. 1. Participants' responses on all measures are summarised in Table 2.
For the corrected model, a significant overall MANOVA effect was observed for the overall diagnosis (Hotelling's $T: F=4.23, p<0.001)$. Follow-up analyses showed significant overall effects for the domains of Psychomotor Speed $\left(F_{3,247}=11.37, p<0.001\right)$, Mental Flexibility $\left(F_{3,247}\right.$ $=7.46, p<0.001)$, Sustained Attention $\left(F_{3,247}=9.37, p<\right.$ $0.001)$, Set Shifting $\left(F_{3,247}=4.34, p=0.005\right)$, Phonemic Fluency $\left(F_{3,247}=7.09, p<0.001\right)$ and Semantic Fluency $\left(F_{3,247}=3.90, p=0.009\right)$.

Post-hoc pairwise comparisons indicated that the ASD group showed the greatest level of EF impairments. The ASD group performed significantly worse compared to at least one or more of the other groups on the domains of: Psychomotor Speed (ASD $<$ EP, ASD $<$ SAD and ASD $<$ TYP); Mental Flexibility (ASD $<$ TYP and ASD $<$ SAD); 


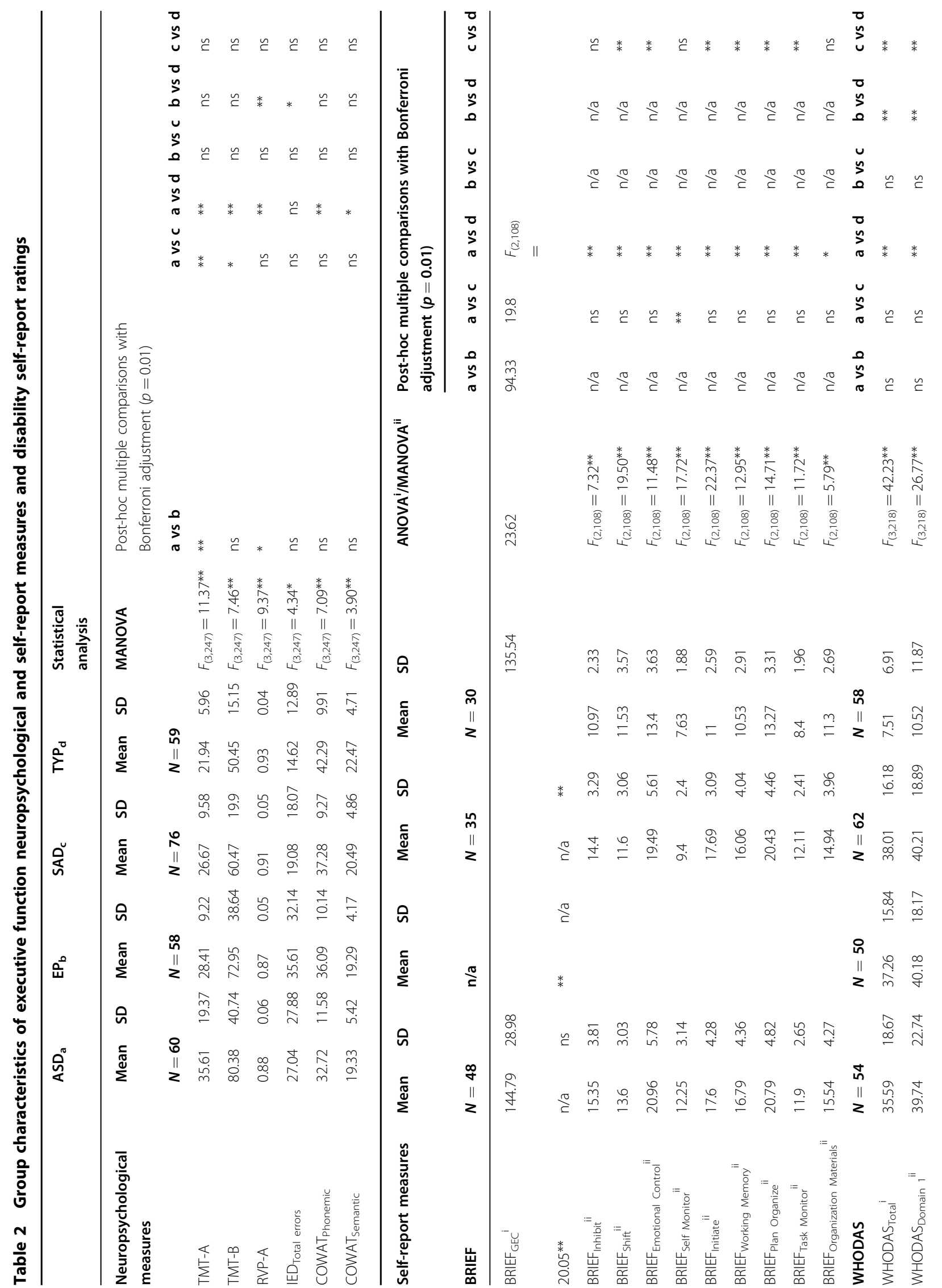




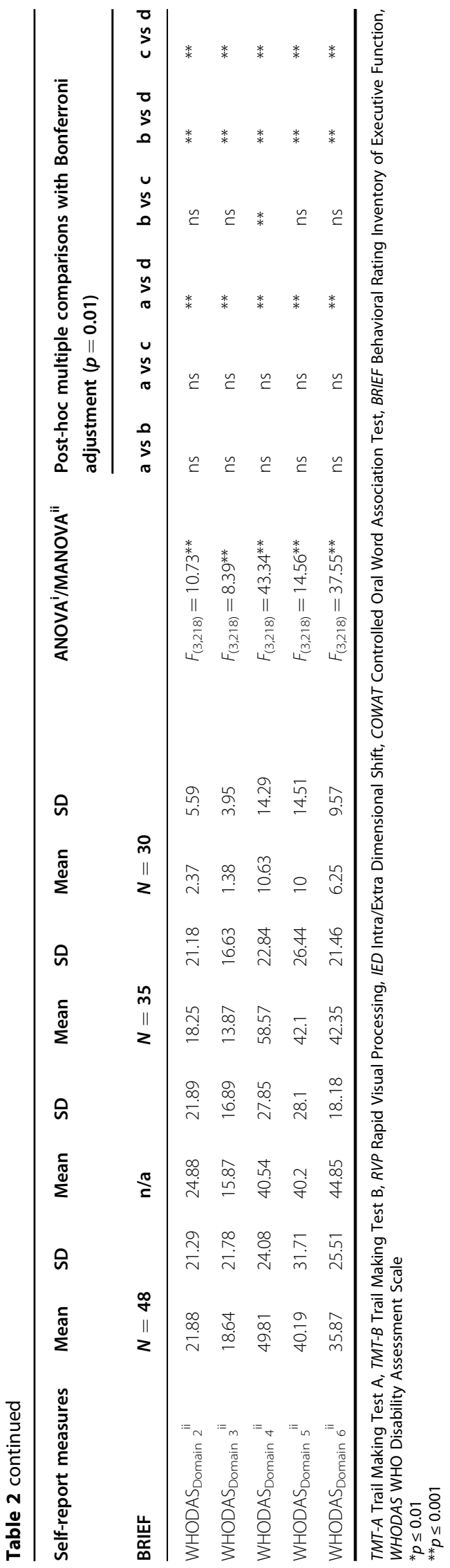

Sustained Attention (ASD < TYP); Phonemic Fluency (ASD < TYP) and Semantic Fluency (ASD < TYP). The EP group was significantly impaired on Sustained Attention $(\mathrm{EP}<\mathrm{ASD}, \mathrm{EP}<\mathrm{TYP})$ and on Set Shifting $(\mathrm{EP}<\mathrm{TYP})$. The SAD group showed intact EF on all performance measures.

The ANOVA analysis comparing differences between groups on the BRIEF (overall score) was significant $\left(F_{2,108}\right.$ $=20.05, p<0.001$ ). Pairwise comparisons revealed that both ASD and SAD groups reported similar and significantly higher levels of EF impairment compared to the TYP group. The overall MANOVA analysis for Diagnosis was significant for the BRIEF clinical scales (Hotelling's $T$ : $F=5.63, p<0.001)$. Pairwise comparisons showed that the ASD group reported significantly impaired EF on all clinical scales compared to the TYP group and on the Self-Monitor subscale compared to the SAD group. The SAD group also reported significant impairment on most clinical scales compared to the TYP group. The nonsignificant results were for the scales of Inhibit, SelfMonitor and Organization of Materials.

A significant overall ANOVA effect was observed for Diagnosis for the WHODAS (overall score) $\left(F_{3,218}=\right.$ 42.23, $p<0.001)$. Pairwise comparisons indicated that each of the clinical groups reported significantly higher levels of overall disability compared to the TYP group with no differences between the clinical groups. The overall MANOVA analysis of the WHODAS domains was also significant (Hotelling's $T: F=10.71, p<0.001$ ). Each of the clinical groups reported significant impairment compared to the TYP group for each of the six WHODAS domains, in addition the SAD group demonstrated significantly higher levels of impairment on the 'Getting along' domain (Domain 4) compared to the EP group.

\section{Effects of EF neuropsychological predictors on disability}

The first MR examined the relationship of the predictors of Diagnosis, EF performance measures, IQ, Education and Depression on disability (Table 3). The model for EF performance measures and disability was significant across the study cohort $\left(F_{10,209}=37.11, p<\right.$ 0.001 ) and explained $64.0 \%$ of the total variance, with significant predictors Education $(\beta=-0.169, p<0.001)$ and Depression $(\beta=0.726, p<0.001)$. As Diagnosis was not a significant predictor no follow up interaction models were examined.

\section{Additive effect of EF self-report predictor on disability}

A second regression analysis was completed for the participants that completed the self-report measure of EF (excludes EP group). The procedure outlined above was followed for Step 1 and the self-report measure (BRIEF) was entered in Step 2 (Table 4). Model 1 accounted for $64.0 \%$ of the total variance $\left(F_{10,97}=17.22, p<0.001\right)$ with 
Table 3 Effects of EF neuropsychological predictors on disability

\begin{tabular}{llll}
\hline & $\boldsymbol{B}$ & SE $\boldsymbol{B}$ & $\boldsymbol{\beta}$ \\
\hline Constant & 49.673 & 20.081 & \\
Diagnosis & 0.662 & 0.882 & 0.036 \\
Education & -1.521 & 0.414 & $-0.169^{* *}$ \\
IQ & 0.044 & 0.112 & 0.020 \\
TMT-A & 0.176 & 0.088 & 0.114 \\
TMT-B & -0.035 & 0.035 & -0.057 \\
RVP-A & -25.174 & 19.108 & -0.071 \\
IED & 0.010 & 0.037 & 0.012 \\
Fluencyl errors $_{\text {Phonemic }}$ & -0.068 & 0.100 & -0.037 \\
Fluency $_{\text {Semantic }}$ & -0.093 & 0.209 & -0.023 \\
DASS $_{\text {Depression }}$ & 1.078 & 0.068 & $0.726^{* *}$ \\
\hline
\end{tabular}

${ }^{* *} p<0.001$

the only significant predictor Depression $(\beta=0.726, p<$ 0.001). When the BRIEF was entered in Step 2 (method Enter), Model 2 accounted for an additional $17.0 \%$ of the variance $\left(F_{1,96}=86.14, p<0.001\right)$ with the following significant predictors: Diagnosis $(\beta=0.583, p<0.001)$, Education $(\beta=0.161, p=0.009)$, IQ $(\beta=0.227, p<0.001)$, $\mathrm{IED}_{\text {Total errors }}(\beta=-0.146, p=0.007)$, Depression $(\beta=$ $0.325, p<0.001)$ and BRIEF $(\beta=0.967, p<0.001)$. A follow-up analysis was performed to examine the interaction of diagnosis with the five significant predictors $($ Diagnosis $\times$ Education, Diagnosis $\times \mathrm{IQ}$, Diagnosis $\times$ IED-

Total errors, Diagnosis $\times$ Depression and Diagnosis $\times$ BRIEF). Depression was a significant predictor of disability for the ASD $(B=0.621, p<0.001)$ and the $\operatorname{SAD}(B=0.711, p<0.001)$ groups. The BRIEF significantly predicted disability for the ASD group only $(B=0.248, p=0.001)$. None of the other interaction comparisons were significant.

\section{Discussion}

This study is the first to compare underlying cognitive markers for three disorders characterised by social impairment, EP, SAD, and ASD, and specifically evaluate $\mathrm{EF}$ and its role in predicting disability. Our study showed that, despite no apparent impairments in intellectual function, ASD participants showed significant and broad impairments across the domains of Mental Flexibility, Sustained Attention and Fluency, as well as Psychomotor Speed. Participants with EP showed impairments on Sustained Attention and Set Shifting. Interestingly, despite adequate EF performance on the objective measures, SAD participants self-reported EF impairment that was of a similar degree to those diagnosed with ASD. Our findings suggest that different cognitive-circuitry is associated with social impairment in these cohorts and in
Table 4 Additive effect of EF self-report predictor on disability

\begin{tabular}{|c|c|c|c|}
\hline & $B$ & SE $B$ & $\beta$ \\
\hline \multicolumn{4}{|l|}{ Step 1} \\
\hline Constant & 49.673 & 29.476 & \\
\hline Diagnosis & 0.662 & 1.295 & 0.036 \\
\hline Education & -1.521 & 0.607 & -0.169 \\
\hline IQ & 0.044 & 0.164 & 0.020 \\
\hline TMT-A & 0.176 & 0.129 & 0.114 \\
\hline TMT-B & -0.035 & 0.052 & -0.057 \\
\hline RVP-A & -25.174 & 28.048 & -0.071 \\
\hline IED & 0.010 & 0.055 & 0.012 \\
\hline Fluency Phonemic & -0.068 & 0.147 & -0.037 \\
\hline Fluency $_{\text {Semantic }}$ & -0.093 & 0.306 & -0.023 \\
\hline DASS Depression & 1.078 & 0.099 & $0.726^{* *}$ \\
\hline \multicolumn{4}{|l|}{ Step 2} \\
\hline Constant & -119.902 & 28.223 & \\
\hline Diagnosis & 10.578 & 1.426 & $0.583^{* *}$ \\
\hline Education & 1.456 & 0.547 & $0.161^{*}$ \\
\hline IQ & 0.500 & 0.130 & $0.227^{* *}$ \\
\hline TMT-A & -0.152 & 0.101 & -0.099 \\
\hline TMT-B & 0.073 & 0.039 & 0.120 \\
\hline RVP-A & -16.501 & 20.490 & -0.046 \\
\hline IED & -0.117 & 0.042 & $-0.146^{*}$ \\
\hline Fluency Phonemic & -0.286 & 0.110 & -0.155 \\
\hline Fluencysemantic & -0.188 & 0.224 & -0.047 \\
\hline DASS Depression $_{\text {. }}$ & 0.482 & 0.097 & $0.325^{* *}$ \\
\hline BRIEF & 0.585 & 0.063 & $0.967^{* *}$ \\
\hline
\end{tabular}

${ }^{*} p<0.01,{ }^{* *} p<0.001$

particular, broad EF connectivity ${ }^{19}$ in ASD, attentional switching ${ }^{17}$ in EP and self-referential processes ${ }^{28}$ in SAD. Further research is now required linking the neurobiological underpinnings of these to this disability.

The second aim of this study was to establish which EF measures predicted disability. Results showed that the selfreport measure of $\mathrm{EF}$ and depression predicted disability overall. The relationship between depression and disability was significant across both the ASD and SAD groups however, the self-report measure of EF explained additional proportion of the disability variance for the ASD group only. This study therefore provides evidence that deficits in EF are particularly pronounced in ASD and may be particularly important in terms of predicting disability.

For the ASD participants, the majority of EF performance measures differentiated these participants from the 
TYP group. While we acknowledge there was variability even within the ASD group itself, this study supports assertions of broad executive dysfunction in young adults with ASD that are likely neuro-developmentally driven. We have previously argued that broad connectivity models of brain development, rather than region specific brain processes, underpin these impairments ${ }^{18}$. Interestingly, the EP group performed significantly worse than the TYP group on the RVP and IED, tests which purport to assess attentional processes ${ }^{30}$. This finding is in line with involvement of the DLPFC ${ }^{31}$. These domains have previously been shown to predict conversion of clinical high risk individuals to psychosis ${ }^{32}$ and may reflect a higher level of impairment. The lack of any further EF impairment in this group supports empirical findings that broad $E F$ in early stages of psychosis remains intact ${ }^{33}$. There was no evidence of EF impairment in SAD on neuropsychological measures, which was expected given that the underlying aetiology for SAD is focused on fear circuits ${ }^{25}$ and maladaptive cognitions ${ }^{27}$.

While we did not have data available for EP participants, both SAD and ASD participants reported similar and significant impairment on the EF self-report measure. This was despite the SAD group showing objective performance on all EF measures that was similar to neurotypical controls. A number of factors may contribute to this finding. Firstly, it may extend the adverse influence of maladaptive cognitions $^{34}$ and self-focused attention ${ }^{35}$ beyond social performance to other areas of self-evaluation, including EF. That is, individuals with SAD negatively evaluate their own adaptive functioning in relation to EF. This might suggest that self-report measures of EF are significantly influenced by anxiety driven biases and exploration of the role of anxiety across all of these cohorts on EF self-report measures is required. Secondly, it may reflect the assertion that self-report ratings of EF assess a different cognitive construct that is not constrained by performance outcomes of objective cognitive measures, but instead is goal oriented and reflects the individual's beliefs relevant to these goals ${ }^{36}$. This may be particularly pertinent in individuals with SAD given their intact performance on objective measures of EF and may augment reporting of executive dysfunction in ASD individuals. Partial support for this proposal was found by the observed low correlation between BRIEF domains and neuropsychological measures of $\mathrm{EF}^{37}$ (Supplementary Table S2), suggesting they might tap different cognitive processes. Alternatively, rating measures of EF may be more ecologically valid and thus better capture functional outcomes associated with $\mathrm{EF}^{7}$.

All of our young adult clinical groups presented to our specialist assessment clinics and reported significant disability compared to the neurotypical control group as measured by the WHODAS. Results showed that in addition to 'Depression', the self-report measure of EF was the strongest predictor of disability in this cohort but, within groups, this relationship was significant only for the ASD group. In contrast, neuropsychological measures of EF were largely unrelated to disability in all groups. The lack of relationship between EF and disability in the other clinical groups indicates that other factors may be more important including affective states ${ }^{24}$ and co-morbid conditions ${ }^{38}$. Further research is needed to elucidate the specific contribution of these factors to disability, which ultimately is linked to participation in society, educational and vocational outcomes ${ }^{3,39}$.

Our study has several limitations. Our performance measures of EF although broad did not encompass all commonly accepted subdomains of EF. We do note, however, our previous work that has highlighted the relative equivalence of EF domains in ASD and across development ${ }^{18}$. Further, the BRIEF was only completed for part of our cohort (ASD/SAD/TYP). Although our results on the relationship between the BRIEF and disability are quite robust, they rely on a self-report measure of disability. Further examination of these against objective measures of disability and on larger samples would further inform the significance of these findings.

\section{Conclusions}

This is the first study to examine both objective and subjective markers of EF across three clinical groups of young adults with social impairment and to evaluate their relationship to disability. The study lends support to the importance of executive dysfunction in the ASD population both in differentiating between clinical groups and in self-reported EF predicting disability. It also indicates that EF deficits are not likely primary contributors to disability for SAD and EP, despite the SAD group reporting EF impairment on self-report measures and the EP group performing worse on tests of sustained attention and attentional shifting. The EF outcomes across our cohort support impaired self-referential processing that may relate to DMN processes in SAD, broad executive dysfunction that may be primarily driven by aberrant connectivity in ASD and, impaired attentional processes driven by the DLPFC in EP. Overall, these findings have treatment implications for young adults with social impairment and suggest that ASD therapeutic support may need to include cognitive training of $E F$, whereas in SAD and EP focus on maladaptive cognitions and attentional processes respectively, may be more appropriate.

\section{Acknowledgements}

We would like to thank Caro Badcock for statistical support. We acknowledge NHMRC postgraduate scholarship to E.A.D. (GNT1056587), an NHMRC

Australian Fellowship (APP 511921) to I.H., and ARC Linkage Project grants (LP110100513; LP110200562), a National Health and Medical Research Council 
Career Development Fellowship (APP1061922), and a Project Grant (1043664; 1125449) to A.J.G.

\begin{abstract}
Author details
${ }^{1}$ Autism Clinic for Translational Research, Brain and Mind Centre, Central Clinical School, Faculty of Medicine, University of Sydney, Camperdown 2050, Australia. ${ }^{2}$ Youth Mental Health Unit, Brain and Mind Centre, Central Clinical School, Faculty of Medicine, University of Sydney, Camperdown 2050, Australia. ${ }^{3}$ School of Psychology, University of Sydney, Camperdown 2050, Australia
\end{abstract}

\section{Conflict of interest}

I.H. is a Commissioner in Australia's new National Mental Health Commission from 2012. He was a director of headspace: the national youth mental health foundation until January 2012. He was previously the chief executive officer (till 2003) and clinical adviser (till 2006) of beyondblue, an Australian National Depression Initiative. He is the Co-Director, Health and Policy at the Brain and Mind Centre that operates two early-intervention youth services under contract to headspace. He has led a range of community-based and pharmaceutical industry-supported depression awareness and education and training programs. He has led projects for health professionals and the community supported by governmental, community agency and pharmaceutical industry partners (Wyeth, Eli Lily, Servier, Pfizer, AstraZeneca) for the identification and management of depression and anxiety. He has received honoraria for presentations of his own work at educational seminars supported by a number of non-government organisations and the pharmaceutical industry (including Servier, Pfizer, AstraZeneca and Eli Lilly). He is a member of the Medical Advisory Panel for Medibank Private and also a Board Member of Psychosis Australia Trust. He leads an investigator-initiated study of the effects of agomelatine on circadian parameters (supported in part by Servier) and has participated in a multicentre clinical trial of the effects of agomelatine on sleep architecture in depression and a Servier-supported study of major depression and sleep disturbance in primary care settings. The other authors declare that they have no conflict of interest.

\section{Publisher's note}

Springer Nature remains neutral with regard to jurisdictional claims in published maps and institutional affiliations.

Supplementary Information accompanies this paper at (https://doi.org/ 10.1038/s41398-018-0193-8).

Received: 28 May 2018 Accepted: 8 June 2018

Published online: 24 September 2018

\section{References}

1. Stein, M. B. \& Kean., Y. M. Disability and quality of life in social phobia: epidemiologic findings. Am. J. Psychiatry 157, 1606-1613 (2000).

2. Wiersma, D. et al. Social disability in schizophrenia: its development and prediction over 15 years in incidence cohorts in six European centres. Psychol. Med. 30, 1155-1167 (2000).

3. Howlin, P. \& Magiati, I. Autism spectrum disorder: outcomes in adulthood. Curr. Opin. Psychiatry 30, 69-76 (2017).

4. Lieb, R. W. \& Bohnert, A. M. Relations between executive functions, social impairment, and friendship quality on adjustment among high functioning youth with autism spectrum disorder. J. Autism Dev. Disord. 47, 2861-2872 (2017).

5. Fujii, Y. et al. Severity of generalized social anxiety disorder correlates with low executive functioning. Neurosci. Lett. 543, 42-46 (2013).

6. Lezak, M., Howieson, D., Bigler, E. \& Tranel, D. Neuropsychological Assessment. (Oxford University Press, New York, 2012).

7. Kenworthy, L., Yerys, B. E., Anthony, L. G. \& Wallace, G. L. Understanding executive control in autism spectrum disorders in the lab and in the real world. Neuropsychol. Rev. 18, 320-338 (2008).

8. Nelson, E. E., Jarcho, J. M. \& Guyer, A. E. Social re-orientation and brain development: an expanded and updated view. Dev. Cogn. Neurosci. 17(Supplement C), 118-127 (2016).
9. Carpenter, W. T. The RDoC controversy: alternate paradigm or dominant paradigm. Am. J. Psychiatry 173, 562-563 (2016).

10. McGrath, L. M. et al. Extending the 'cross-disorder' relevance of executive functions to dimensional neuropsychiatric traits in youth. J. Child Psychol. Psychiatry 57, 462-471 (2016).

11. Hickie, I. B. et al. Evaluating differential developmental trajectories in to adolescent-onset mood and psychotic disorders. BMC Psychiatry 13, 303 (2013).

12. Lee, R. S. C. et al. Neuropsychological and socio-occupational functioning in young psychiatric outpatients: a longitudinal investigation. PLOS ONE 8, e58176 (2013).

13. Leung, R. C., Vogan, V. M., Powell, T. L., Anagnostou, E. \& Taylor, M. J. The role of executive functions in social impairment in autism spectrum disorder. Child Neuropsychol. 22, 336-344 (2016).

14. Woon, P. S., Chia, M. Y., Chan, W. Y. \& Sim, K. Neurocognitive, clinical and functional correlates of subjective quality of life in Asian outpatients with schizophrenia. Prog. Neuro-Psychopharmacol. Biol. Psychiatry 34, 463-468 (2010).

15. Fu, S., Czajkowski, N., Rund, B. R. \& Torgalsbøen, A.-K. The relationship between level of cognitive impairments and functional outcome trajectories in firstepisode schizophrenia. Schizophr. Res. 190, 144-149 (2017).

16. Eisenberg, D. P. \& Berman, K. F. Executive function, neural circuitry, and genetic mechanisms in schizophrenia. Neuropsychopharmacology 35, 258-277 (2009).

17. Wood, S. J. et al. Progressive changes in the development toward schizophrenia: studies in subjects at increased symptomatic risk. Schizophr. Bull. 34 322-329 (2008).

18. Demetriou, E. A. et al. Autism spectrum disorders: a meta-analysis of executive function. Mol. Psychiatry. 23, 1198-1204 (2017).

19. Libero, L. E., DeRamus, T. P., Lahti, A. C., Deshpande, G. \& Kana, R. K. Multimodal neuroimaging based classification of autism spectrum disorder using anatomical, neurochemical, and white matter correlates. Cortex $\mathbf{6 6}$ (Supplement C), 46-59 (2015).

20. Yerys, B. E. et al. Neural correlates of set-shifting in children with autism. Autism Res. 8, 386-397 (2015)

21. D'Cruz, A. M., Mosconi, M. W., Ragozzino, M. E., Cook, E. H. \& Sweeney, J. A. Alterations in the functional neural circuitry supporting flexible choice behavior in autism spectrum disorders. Transl. Psychiatry 6, e916 (2016).

22. Wallace, G. L. et al. Real-world executive functions in adults with autism spectrum disorder: profiles of impairment and associations with adaptive functioning and co-morbid anxiety and depression. J. Autism Dev. Disord. 46 1071-1083 (2016).

23. Pugliese, C. E. et al. Longitudinal examination of adaptive behavior in autism spectrum disorders: influence of executive function. J. Autism Dev. Disord. 46, 467-477 (2016)

24. OToole, M. S. \& Pedersen, A. D. A systematic review of neuropsychological performance in social anxiety disorder. Nord. J. Psychiatry 65, 147-161 (2011).

25. Davidson, R. J. Anxiety and affective style: role of prefrontal cortex and amygdala. Biol. Psychiatry 51, 68-80 (2002).

26. Whitfield-Gabrieli, S. et al. Brain connectomics predict response to treatment in social anxiety disorder. Mol. Psychiatry 21, 680-685 (2016).

27. Dryman, M. T., Gardner, S., Weeks, J. W. \& Heimberg, R. G. Social anxiety disorder and quality of life: how fears of negative and positive evaluation relate to specific domains of life satisfaction. J. Anxiety Disord. 38, 1-8 (2016)

28. Cremers, H. R. \& Roelofs, K. Social anxiety disorder: a critical overview of neurocognitive research. Wiley Interdiscip. Rev. Cogn. Sci. 7, 218-232 (2016).

29. Allen, P., Bennett, K. P. \& Heritage, B. SPSS Statistics Version 22: A Practical Guide. 3rd edn, (Cengage Learning Australia, South Melbourne, Victoria 2014).

30. De Luca, C. R. et al. Normative data from the CANTAB. I: development of executive function over the life span. J. Clin. Exp. Neuropsychol 25, 242-254 (2003).

31. Silton, R. L. et al. The time course of activity in dorsolateral prefrontal cortex and anterior cingulate cortex during top-down attentional control. NeuroImage 50, 1292-1302 (2010).

32. Seidman, L. J. et al. Association of neurocognition with transition to psychosis: baseline functioning in the second phase of the North American prodrome longitudinal study. JAMA Psychiatry 73, 1239-1248 (2016). 
33. Ayesa-Arriola, R. et al. Diagnosis and neurocognitive profiles in first-episode non-affective psychosis patients. Eur. Arch. Psychiatry Clin. Neurosci. 266 619-628 (2016).

34. Goldin, P. R. et al. Impact of cognitive behavioral therapy for social anxiety disorder on the neural dynamics of cognitive re-appraisal of negative selfbeliefs. JAMA Psychiatry 70, 1048-1056 (2013).

35. Boehme, S., Miltner, W. H. R. \& Straube, T. Neural correlates of selffocused attention in social anxiety. Soc. Cogn. Affect. Neurosci. 10, 856-862 (2015).

36. Toplak, M. E., West, R. F. \& Stanovich, K. E. Practitioner review: do performancebased measures and ratings of executive function assess the same construct? J. Child Psychol. Psychiatry 54, 131-143 (2013).
37. Toplak, M. E., Bucciarelli, S. M., Jain, U. \& Tannock, R. Executive functions: performance-based measures and the Behavior Rating Inventory of Executive Function (BRIEF) in adolescents with Attention Deficit/Hyperactivity Disorder (ADHD). Child Neuropsychol. 15, 53-72 (2008).

38. Moss Lowengrub, K. Stryjer, R., Birger, M. \& lancu, I. Social anxiety disorder comorbid with schizophrenia: the importance of screening for this underrecognised and undertreated condition. Isr. J. Psychiatry Relat. Sci. 52, 40-46 (2015).

39. van Heijst, B. \& Geurts, H. Quality of life in autism across the lifespan: a metaanalysis. Autism 19, 158-167 (2015). 